\title{
Features of the energy spectrum of indium antimonide quantum dots
}

\author{
A. I. Mikhailov, V. F. Kabanov, N. D. Zhukov, E. G. Glukhovskoy \\ Saratov State University, Department of Nano- and Biomedical Technologies, \\ Astrakhanskaya, 83, Saratov, 410012, Russia \\ glukhovskoy@gmail.com
}

PACS 73.22.f, 73.21.La

DOI 10.17586/2220-8054-2017-8-5-596-599

\begin{abstract}
Energy spectrum of indium antimonide (InSb) quantum dots (QD) was analyzed in this paper. Properties of energy spectrum levels were determined both by calculations and experiments using differential tunneling current-voltage characteristics (CVC) rated to static conductivity. It was confirmed that relatively large QD (size of about 20-25 nm) exhibit quantum size effects. Critical values of the characteristic parameters of InSb QD are analyzed, in which application of the differential tunneling current-voltage characteristics method for express analysis of the characteristic sizes of QD leads to significant errors (more than $10 \%$ ).
\end{abstract}

Keywords: quantum dots, indium antimonide, differential tunneling current-voltage characteristics, energy spectrum.

Received: 17 July 2017

Revised: 5 August 2017

\section{Introduction}

Quantum dots QD are among the most interesting quantum-sized structures in terms of application in devices for nanoelectronics and optoelectronics (ultrafast transistors, light-emitting diodes, lasers, photoelectric converter cells). Several of the semiconductor compounds $A_{3} B_{5}$ are promising materials for these purposes due to its energy spectrum features and extremely low effective mass values for conduction electrons. Low values of effective mass provide high De Broglie wavelength for conduction electrons that is tens of nanometers. In this regard, specific phenomena caused by dimensional quantization of the energy spectrum of electrons can be observed in relatively large structures, primarily in InSb samples, that is a narrow-band semiconductor with record values of the effective mass of electrons in the conduction band $[1,2]$. This makes it possible to substantially simplify the experimental studies and reduce the cost of technologies for the development of corresponding nanoelectronic structures.

\section{Materials and methods}

Indium antimonide QD samples were obtained by a technique similar to that used in $[3,4] .0 .0014 \mathrm{~g}$ of InSb powder $\left(M_{r}=236.578 \mathrm{~g} /\right.$ mole $)$ was dispersed in $1 \mathrm{ml}$ of chloroform $\left(C_{(\mathrm{InSb})}=6 \mathrm{mM}\right)$ followed by ultrasonic treatment for $30 \mathrm{~min}$. Suspension of colloid nanoparticles was mixed with Arachic acid solution with an initial concentration of $1 \mathrm{mM}$ in a volume ratio of 1:1. Resulted suspension was used to form a Langmuir monolayer on the surface of deionized water. It was injected onto the surface of the water, kept for 10 minutes until complete evaporation of chloroform and then the monolayer was condensed and transferred the solid support by the Langmuir-Schaeffer method. Surface pressure during transfer was $20 \mathrm{mN} / \mathrm{m}^{2}$ and it was kept constant. A glass slide with a conductive layer of indium-tin oxide (ITO) on the surface was used as the support. After complete drying under normal conditions, the obtained films were investigated with scanning electron microscopy (SEM) and scanning tunneling microscopy (STM).

\section{Results and discussion}

Tunneling of electrons from the ITO electrode into the probe of the tunneling microscope occurs by means of discrete levels of QD. Model representations of this process considered in the work of the authors [5] and shown on the Fig. 1 $(\mathrm{a}, \mathrm{b})$ under applied voltages $V_{1}$ and $V_{2}$, corresponding to tunneling through the 1 st and 2 nd electron energy levels of the discrete spectrum of QD.

In the case of quantum dots, the energy spectrum has a discrete nature, and the density of electronic states $g(\varepsilon)$ is described by the Dirac $\delta$-function and theoretically it is a set of infinitesimally narrow and high peaks:

$$
g(\varepsilon)=\sum_{i} \delta\left(\varepsilon-\varepsilon_{i}\right)
$$

An exact calculation of the function $g(\varepsilon)$ is difficult in general, since the isoenergetic surfaces have a rather complex shape. However the problem usually reduces to solving the Schrödinger equation for a free particle with 


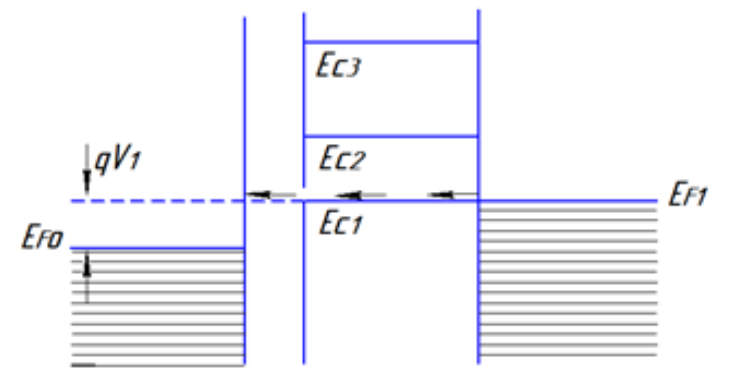

(a)

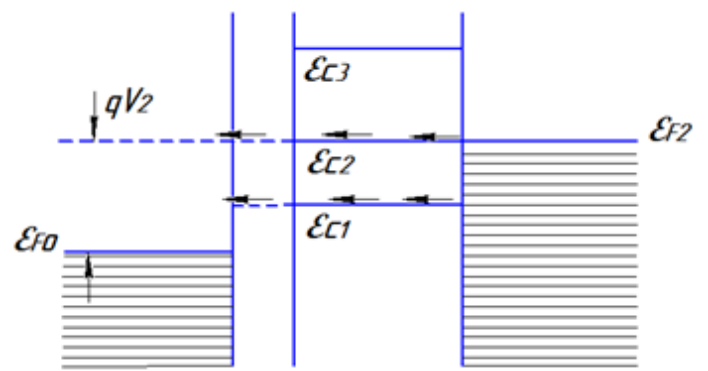

(b)

FIG. 1. Tunneling of electrons through a quantum dot

an effective mass $m^{*}$ by the effective mass approximation [6]. Another approximation is the use of energy bands with a quadratic dispersion law. This is permissible in the case of small pulses and small electron energies, which is realized in the tasks under consideration.

When QD model of a cubic shape with edge $d$ is used:

$$
\varepsilon_{i}=\frac{(\pi \hbar)^{2}}{2 m^{*}} \cdot \frac{1}{d^{2}} \cdot\left(l^{2}+m^{2}+n^{2}\right),
$$

where $l, m, n=1,2,3, \ldots$ are positive natural numbers corresponding to the sub bands numbers; $m^{*}$ is the effective mass of the electron, and $d$ is the characteristic size of the quantum dot.

In case of a QD of a spherical shape with the radius $d$ in the one-electron spectrum in a parabolic well approximation [7]:

$$
\varepsilon_{i}=\frac{(\pi \hbar)^{2}}{2 m^{*}} \cdot \frac{1}{d^{2}} \cdot(4 n+2 l+3)
$$

where $n$ is the radial quantum number $(n=0,1,2, \ldots) ; l$ is the orbital quantum number $(l=0,1,2, \ldots)$.

Calculated values of the electron energy for the first three allowed energy levels $\left(\varepsilon_{1}, \varepsilon_{2}, \varepsilon_{3}\right)$ in accordance with (4), (5) for InSb are shown in Fig. 2(a, b). The value of the effective mass of electrons in the conduction band of InSb is used in the calculations: $m^{*}=0.013 m_{0}$, where $m_{0}$ is the mass of a free electron. Eqs. (2) and (3) (Fig. 2) imply that, as the QD size decreases, not only the "effective" width of the band-gap will increase, but also the energy distance between the allowed energy levels in the conduction band will increase. Presented in the [8] experimental results for InSb quantum dots with size $3 \pm 7 \mathrm{~nm}$ confirming these conclusions.

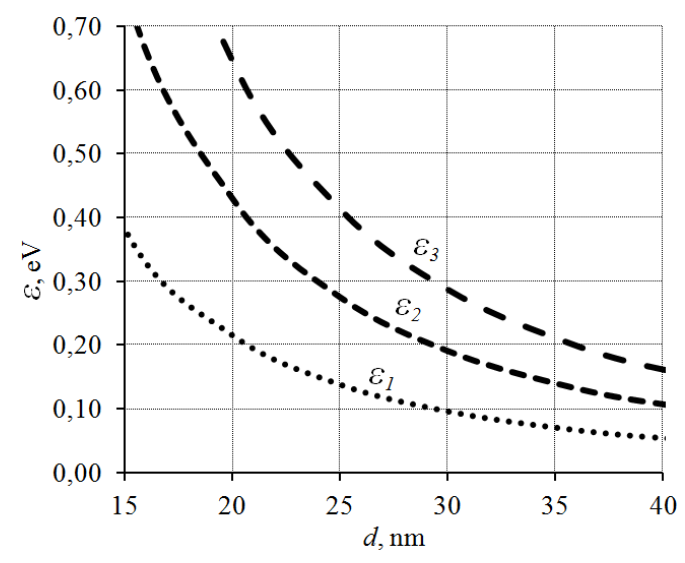

(a)

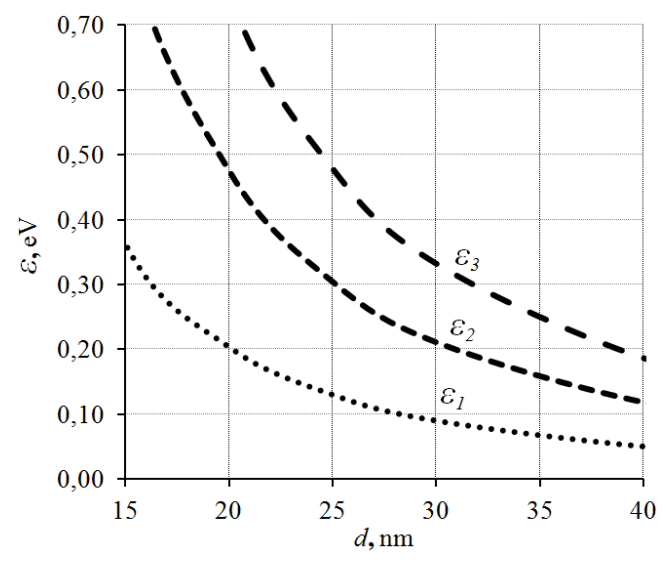

(b)

FIG. 2. Calculated values of the electron energy for the first allowed levels of QD InSb as a function of the characteristic size of the nanoobject $d$ : (a) - according to the cubic QD model, (b) - according to the spherical QD model

Electron scattering leads to broadening of the energy levels $\Delta \varepsilon_{i}$ and limiting the amplitude of the peaks [9,10]: $\Delta \varepsilon_{i} \propto \hbar / \tau$, where $\tau$ is lifetime of an electron in quasi stationary states of QD. 
Broadening of this kind in real QD occurs due to the finite lifetime of charge carriers caused by spontaneous emission, interaction with phonons, and a number of other processes [9,11].

The magnitude of the broadening $\Delta \varepsilon_{i}$ is related to the value of $\varepsilon_{i}$. Both these parameters affect the form and nature of $g(\varepsilon)$.

The nature and the degree of the broadening $\Delta \varepsilon_{i}$ in accordance with representations of the authors [9] in case of simple energy bands with a quadratic dispersion law for the spherical QD model can be represented by the following equation according to the corresponding approximations:

$$
\Delta \varepsilon_{i} \approx 16 \frac{\varepsilon^{1 / 2}}{\varepsilon_{i}}\left(\varepsilon_{i}-\varepsilon\right)^{3 / 2} \cdot e^{-2 \Delta r\left(\frac{2 m^{*}\left(\varepsilon_{i}-\varepsilon\right)^{1 / 2}}{\hbar}\right)},
$$

where $\Delta r$ is the thickness of the QD shell.

The value $\varepsilon_{i}$ is mainly determined by the characteristic size of QD $d$ and hence by the technology of the synthesis.

SEM and optical spectroscopy methods do not provide correct interpretation of the experimental data if structure of the quantum particle is not homogeneous or several particles form a conglomerate. In this regard, STM method was additionally used to analyze the features of the electronic spectrum of the obtained InSb QDs films. Investigations were carried out with the scanning probe microscope SPM Nanoeducator II by the described methods $[1,10]$.

Localized energy levels $\varepsilon_{i}$ associated with the properties of a quantum-dimensional object can be observed in differential tunneling CVC rated to static conductivity as individual peaks. To analyze the experimental tunneling CVC the dependence of $(d I / d V) /(I / V)$ on the voltage $V$ was used in analogy with the approaches described in $[1,10,12]$, since the method of normalized differential CVC is more informative. Typical results of measurements are shown in Fig. 3. Statistical processing of the values of the first three levels determined the following energies: $\varepsilon_{1} \sim(0.15-0.20) \mathrm{eV}, \varepsilon_{2} \sim(0.30-0.35) \mathrm{eV}, \varepsilon_{3} \sim(0.55-0.65) \mathrm{eV}$ (an error is on the level of $\left.k T\right)$. These values correspond to the characteristic size of the obtained QDs of 20-25 nm. It should be noticed that the difference between the cubic and spherical models did not exceed $8 \%$.

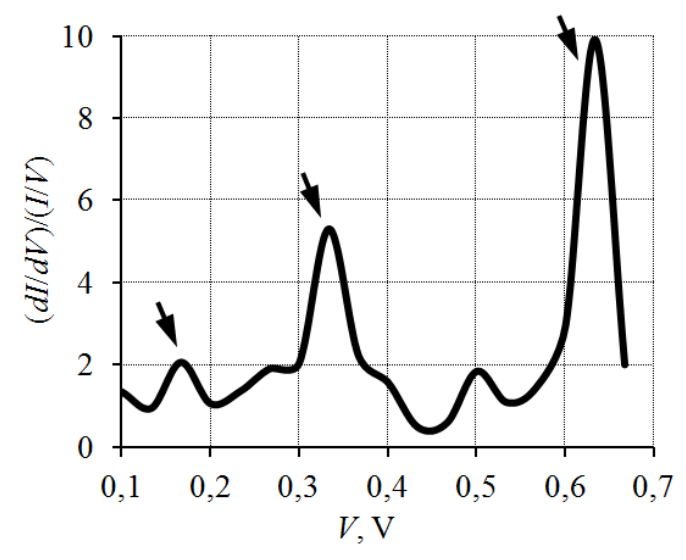

FIG. 3. Rated differential current-voltage characteristics of InSb QD

The value $\Delta \varepsilon_{i}$ should be taken into account in analysis of the experimental results for estimating the electron spectrum of QD by the method of rated differential tunneling CVC: the peaks corresponding to $\varepsilon_{i}$ can be "smoothed". In our opinion, this express analysis method of the characteristic size of QD is difficult at a value of $\Delta \varepsilon_{i}$ more than $10 \%$. Obviously, described broadening will be the most significant for the first level of QD, measured from the bottom of the conduction band for the bulk material. In this paper, we estimate the value $\Delta \varepsilon_{1}$ in accordance with Eq. (6), depending on the characteristic size of the nanoobject $d$. During the calculations, the value of the conduction electron kinetic energy was chosen on the level of $3 / 2 k T$. The results are shown in Fig. 4.

The results of the calculations show that at QD shell thickness of about $1 \mathrm{~nm}$ (particles close to "open QD") the magnitude of the broadening of the first energy level of the InSb QD becomes significant at the characteristic size $d$ less than $10 \mathrm{~nm}$. Errors of the of differential tunneling CVC method for express analysis of the QD characteristic size are significant (more than $10 \%$ ) in this case. If QD shell thickness is increased the magnitude of the energy level broadening from the characteristic size $d$ of the quantum-dimensional object is substantially reduced. However in this case the lifetime of the charge carriers in the inner region of the QD, and, correspondingly, the value of $\Delta \varepsilon_{i}[9]$, will vary. 


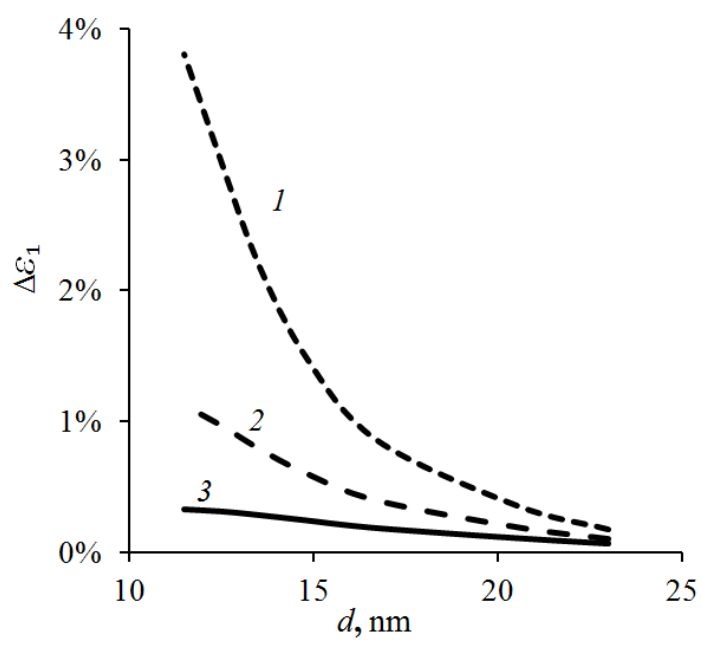

FIG. 4. Calculated values of the broadening of the 1st energy level of the InSb QD spectrum: 1 - QD shell thickness $1 \mathrm{~nm} ; 2$ - QD shell thickness $2 \mathrm{~nm} ; 3$ - QD shell thickness $3 \mathrm{~nm}$

\section{Conclusion}

Thus, several features of the InSb quantum dots energy spectrum was analyzed, taking into account the broadening of the energy spectrum levels. Positions of the levels were determined by processing the experimental tunneling CVC. Estimation of the QD characteristic size was carried out using "cubic" and "spherical" models. Critical values for the characteristic sizes of InSb quantum dots are analyzed, which lead to significant (more than $10 \%$ ) errors for the differential tunneling CVC method for express analysis of the QD characteristic sizes. The obtained results can be used to clarify the understanding of the electronic processes features in the quantumdimensional objects and its physical interpretation.

\section{Acknowledgements}

This work was supported by grants from the Russian Foundation for Basic Research Projects No. 16-07-00093 and No. 16-07-00185.

\section{References}

[1] Mikhailov A.I., Kabanov V.F., Zhukov N.D. Peculiarities of field electron emission from submicron protrusions on a rough InSb surface. Technical Physics Letters, 2015, 41 (11), P. 1065-1067.

[2] Chang A.Y., et al. Carrier Dynamics in Highly Quantum-Confined, Colloidal Indium Antimonide Nanocrystals. ACS Nano, 2014, 8 (8), P. 8513-8519.

[3] Reiss P., Protière M., Li L. Core/shell semiconductor nanocrystals. SMALL, 2009, 5 (2), P. 154-168.

[4] Speranskaya E.S. , Beloglazova N.V., et al. Polymer-coated fluorescent CdSe-based quantum dots for application in immunoassay. Biosensors and Bioelectronics, 2014, 53, P. 225-231.

[5] Mikhailov A.I., Kabanov V.F. Electronic properties of A2B6 semiconductor quantum dots. - In the Collect.: Nano- and biomedical technologies. Quality management. Problems and prospects. Collection of scientific articles. Saratov, SGU Publishing house, 2016,75 p. (in Russian)

[6] Dragunov V.P., Neizwestny I.G., Gridchin V.A. Fundamentals of nanoelectronics. Fizmatkniga, Moscow, 2006,496 p. (in Russian)

[7] Kaputkina, N.E., Lozovik Y.E. "Spherical" quantum dots. Phys. Solid State, 1998, 40 (11), P. 1935-1936.

[8] Wang T., et al. Size-dependent energy levels of InSb quantum dots measured by scanning tunneling spectroscopy. ACS Nano, 2015, 9 (1), P. 725-732.

[9] Zegrya G.G., Samosvat D.M. Carrier energy spectrum and lifetime in quantum dots in electric field. J. Exp. Theor. Phys., 2009, 108 (6), P. 907-916.

[10] Mikhailov A.I., Kabanov V.F., Zhukov N.D. Peculiarities of field electron emission from submicron protrusions on a rough InSb surface. Technical Physics Letters, 2015, 41 (6), P. 568-570.

[11] Korolev N.V., Starodubtsev S.E., Bormontov E.N., Klinskikh A.F. Features of the electron spectrum of an open spherical quantum dot with a delta potential. Condensed media and interphase boundaries, 2011, 13 (1), P. 67-71. (in Russian)

[12] Mikhailov A.I., Kabanov V.F., Gorbachev I.A., Glukhovskoi E.G. A study of specific features of the electronic spectrum of quantum dots in CdSe semiconductor. Technical Physics Letters, 2016, 42 (8), P. 796-798.

[13] Swart I., et al. Scanning probe microscopy and spectroscopy of colloidal semiconductor nanocrystals and assembled structures. Chem. Rev., 2016, 116 (18), P. 11181-11219. 